\title{
Investigation Into Accessible Surface Vinyl Concentrations of Nonstoichiometric PDMS Microspheres from Hydrosilylation Reactions and Their Further Crosslinking Reactions
}

Ma, Baoguang; Hansen, Jens Henrik; Hvilsted, Søren; Skov, Anne Ladegaard

Published in:

Macromolecular Materials \& Engineering

Link to article, DOI:

10.1002/mame.201400406

Publication date:

2015

Document Version

Peer reviewed version

Link back to DTU Orbit

Citation (APA):

Ma, B., Hansen, J. H., Hvilsted, S., \& Skov, A. L. (2015). Investigation Into Accessible Surface Vinyl

Concentrations of Nonstoichiometric PDMS Microspheres from Hydrosilylation Reactions and Their Further Crosslinking Reactions. Macromolecular Materials \& Engineering, 300(6), 627-636.

https://doi.org/10.1002/mame.201400406

\section{General rights}

Copyright and moral rights for the publications made accessible in the public portal are retained by the authors and/or other copyright owners and it is a condition of accessing publications that users recognise and abide by the legal requirements associated with these rights.

- Users may download and print one copy of any publication from the public portal for the purpose of private study or research.

- You may not further distribute the material or use it for any profit-making activity or commercial gain

- You may freely distribute the URL identifying the publication in the public portal 


\title{
Investigation into accessible surface vinyl concentrations of nonstoichiometric PDMS microspheres from hydrosilylation reactions and their further crosslinking reactions
}

\author{
Baoguang $\mathrm{Ma}^{\ddagger}$, Jens Henrik Hansen ${ }^{\S}$, Søren Hvilsted ${ }^{\ddagger}$, Anne Ladegaard Skov ${ }^{{ }^{*}}$ \\ ${ }^{\ddagger}$ Danish Polymer Centre, Department of Chemical and Biochemical Engineering, DTU, DK-2800 Kgs. Lyngby, \\ Denmark \\ ${ }^{\S}$ Maersk Oil Research and Technology Centre, Education City, P.O. Box 210112, Doha, Qatar
}

\begin{abstract}
The introduction of surface vinyl groups to PDMS microspheres broadens the latter's applicability range since the microspheres can be further functionalized or crosslinked into elastomers. Quantification of the surface vinyl concentration of PDMS microspheres is therefore essential. Here, a novel titration method, which is based on efficiently and covalently bonding mono-functional hydride to vinyl, is employed to determine surface vinyl concentration. The titration method exhibits good reproducibility in detection, thereby underlining its potential applicability as a general titration method for cured silicones. Vinyl functional PDMS microspheres are mixed with a hydride crosslinker and non-reactive silicone oil to create a system which allows for extensive crosslinking. Both visual observations and rheological studies show that a robust macroscopic PDMS elastomer is obtained upon crosslinking. Furthermore, the influence of stoichiometric imbalance, and the weight fraction of silicone oil on the terminal storage modulus of the macroscopic PDMS elastomer, is investigated. The wide range of stoichiometries facilitating crosslinking between the PDMS microspheres and the hydride crosslinker found in this study is beneficial for delivering PDMS elastomer to hard-to-reach places, since the sensitivity of the system on concentration fluctuations is small.
\end{abstract}

Keywords: PDMS microspheres, surface vinyl concentration, crosslinking reaction, macroscopic network

\section{Introduction}

PDMS microspheres are attracting a great deal of attention, because not only do they possess unique PDMS properties but, like other polymeric microspheres, they also retain a number of excellent characteristics such as large surface area, high diffusivity and high reactivity ${ }^{1,2}$. The versatility of PDMS microspheres explains their widespread utilisation in research and applications $s^{3,4}$. In the application of smart materials, Peng et al. ${ }^{5}$ used a microfluidic system to prepare monodisperse PDMS microspheres of the order of milligrams. Alternatively, Gonzalez et al. ${ }^{6}$ used emulsion polymerisation to prepare PDMS microspheres with broader size distribution in surfactant solutions, thus allowing for the preparation of large amounts of 
PDMS microspheres which, using either microfluidic system or emulsion polymerisation, is based on the crosslinking reaction between vinyl-terminated PDMS and a hydride crosslinker ${ }^{7}$. By increasing the mixing ratio (stoichiometry) between vinyl-terminated PDMS and a hydride crosslinker, PDMS microspheres with surface vinyl groups can be obtained.

Surface vinyl groups confer PDMS microspheres with surface activity, which includes the potential for further chemical modification on the surface of the PDMS microspheres and an increase in their miscibility with other polymers, thereby broadening their overall applicability. For potential in surface chemical modification, surface vinyl groups allow for grafting a polymeric layer onto the surface of PDMS microspheres, thus rendering them multifunctional ${ }^{8}$. In order to increase miscibility, surface vinyl groups allow for blending incompatible polymers with the PDMS microspheres and thus creating reinforced materials ${ }^{9}$. Both the grafting density of the functional polymeric layer and blending efficiency with the incompatible polymers correlate to surface vinyl concentrations, and therefore it is essential to quantify it accordingly.

Quantifying the surface vinyl concentration of PDMS microspheres is challenging, due to their highly crosslinked structure and hydrophobicity. Common techniques such as ${ }^{1} \mathrm{H}$ NMR spectroscopy and titration (e.g. bromine titration) are not suitable; for example, for ${ }^{1} \mathrm{H}$ NMR measurement, samples need to be completely dissolved in deuterated solvent ${ }^{10}$. However, PDMS microspheres are highly crosslinked and thus not soluble in any solvent. For bromine titration, the reaction between bromine and vinyl groups in aqueous solution is utilised to determine the vinyl concentration of polymeric microspheres ${ }^{11}$. However, the aqueous solution used in this process may alter the surface properties of the PDMS microspheres and extract the vinyl groups from the bulk to the surface, thereby resulting in inaccuracy.

Here, a novel titration method is employed which exploits the way in which mono-functional hydrideterminated PDMS reacts with the surface vinyl groups of the PDMS microspheres through a hydrosilylation reaction at a ratio of $1: 1^{12,13}$. The covalently bonded mono-functional hydride will result in weight increase in the PDMS microspheres, thus allowing for the quantification of surface vinyl concentration. The titration method also takes advantage of the high miscibility between the mono-functional hydride and the PDMS microspheres, such that the latter's surface properties will not be altered and the vinyl groups in the bulk will not be extracted to the surface. Not only is the titration method applicable for PDMS microspheres, to determine surface vinyl concentration, but it can also be utilised in determining this property in other commercial silicone materials, i.e. room-temperature vulcanising silicones and liquid silicone rubbers.

Surface vinyl groups on PDMS microspheres allow for further crosslinking reactions with the hydride crosslinker and thus yield a macroscopic PDMS network. The extent of the crosslinking reaction and the properties of the resulting macroscopic PDMS network are influenced by the surface vinyl concentration of the PDMS microspheres, the amount of hydride crosslinker and the surrounding medium. Surface vinyl concentration, which can be detected by applying the mono-functional titration method, allows for estimating the amount of hydride crosslinker needed in the crosslinking reaction ${ }^{14}$. Both surface vinyl concentration and the amount of the hydride crosslinker determine the density of the inter-particle crosslinking points in the macroscopic PDMS network. Regarding the surrounding medium, non-reactive silicone oil with a low molecular weight is used for the following reasons. First of all, it promotes the diffusion of the hydride crosslinker, allowing it to approach and react with the surface vinyl groups. Secondly, silicone oil with a low molecular weight does not get tangled up with the macroscopic PDMS 
network, since this molecular weight $(2,000 \mathrm{~g} / \mathrm{mol})$ is far below the molecular entanglement weight of PDMS $(12,000 \mathrm{~g} / \mathrm{mol})^{15}$. Accordingly, the properties of the macroscopic PDMS network depend solely on the weight fraction of the silicone oil. The crosslinking reaction between surface vinyl groups and the hydride crosslinker allows for obtaining a macroscopic PDMS network and thus delivering PDMS elastomer to hardto-reach places in an oil reservoir, where elastic seals can be utilised to increase oil production ${ }^{16-18}$.

\section{Experimental section}

\section{Materials}

The chemicals employed were: Sylgard 184 consisting of vinyl-terminated PDMS (Batch A) and a curing agent (Batch B) (RTV silicone elastomer, Dow Corning), 25-35\% (methylhydrosiloxane) with 65\%-70\% (dimethylsiloxane) copolymer (HMS-301) $\left(M_{n}=2,000 \mathrm{~g} / \mathrm{mol}\right.$, 8-functional hydride crosslinker, Gelest), monofunctional hydride (MCR-H21) $\left(M_{n}=4,500 \mathrm{~g} / \mathrm{mol}\right.$, Gelest), platinum-cyclovinylmethylsiloxane complex (SIP6832.2 catalyst) (Gelest), polyvinyl alcohol (PVA) $\left(M_{w}=22,000 \mathrm{~g} / \mathrm{mol}\right.$, Fluka), sodium dodecyl sulphate (SDS) $(>99 \%, B D H)$, non-reactive silicone oil (20cSt, $M_{n}=2,000 \mathrm{~g} / \mathrm{mol}$, Dow Corning) and deionised water.

\section{Apparatus}

Homogeneous mixtures were made by mixing batches A and B of Sylgard 184 using a speed mixer (Synergy Devices Ltd, UK) at room temperature.

The size distribution of PDMS microspheres was measured by Mastersizer (Malvern, UK) in a jar tester. The instrument was equipped with a laser with a wavelength of $633 \mathrm{~nm}$, operational in the size range $1 \mu \mathrm{m}$ to $1000 \mu \mathrm{m}$.

The morphology of the PDMS microspheres was analysed with an optical microscope (Leica, German).

Rheological measurements were performed in an AR2000 stress-controlled rheometer (TA Instruments, US), and measurements were made with a strain of $2 \%$ to ensure they would be within the linear regime of the material as well as to minimise disruption to the network ${ }^{19}$. Similar to other measurements of in situ crosslinking reactions, the frequency in the rheological measurements was set to $1 \mathrm{~Hz}^{20}$. In order to have a moderate crosslinking reaction rate, measuring temperature was set to $50^{\circ} \mathrm{C}^{21}$. All measurements were performed in the presence of a $10 \mathrm{ppm}$ platinum catalyst.

\section{Experimental}

\section{Preparation of vinyl functional PDMS microspheres}

A total of 8g Sylgard 184 vinyl-terminated PDMS (Batch A) and respective amounts of curing agent (Batch B) were mixed in a container to ratios of $15: 1,20: 1$ and 25:1 (recommended ratio is 10:1 according to the manufacturer) by using a speed mixer at 3500rpm for 2 minutes. The mixture was then poured into a conical flask with $200 \mathrm{~g}$ of aqueous surfactant solution, which contained $3 \%(w t)$ SDS and $1 \%(w t)$ PVA. A $2 \mathrm{~cm}$ diameter impeller with two inclined blades was then used to stir the solution for 2 minutes at 2000rpm to produce an emulsion. Upon the formation of the emulsion, the stirring speed was reduced to 500rpm and the system was cured at $80^{\circ} \mathrm{C}$ for 2 hours in a water bath. The PDMS microspheres were then obtained by filtering. To ensure that the crosslinking reaction in the PDMS microspheres was finished, and to remove 
residual water, the PDMS microspheres were dried overnight at $80^{\circ} \mathrm{C}$. The obtained vinyl functional PDMS microspheres were denoted as 15:1 PDMS microspheres, 20:1 PDMS microspheres and 25:1 PDMS microspheres. More details about the preparation procedure can be found in Gonzalez et al. ${ }^{6}$.

Determining the soluble fraction of the vinyl functional PDMS microspheres

Approximately $0.5 \mathrm{~g}$ vinyl functional PDMS microspheres were swollen in heptane for 48 hours (20-30 times excess, i.e. $15-25 \mathrm{~mL}$ solvent to a sample of $0.5 \mathrm{~g}$ ). In all experiments, we ensured that the PDMS microspheres were fully covered in heptane for 48 hours $^{22}$, after which they were separated from the heptane solution. The residual heptane was removed by evaporation over another 48 hours under atmospheric conditions. Afterwards, the dry PDMS microspheres were weighed and the soluble fraction of PDMS microspheres was calculated.

Titration determination of PDMS microsphere surface vinyl concentration

The vinyl concentration of Sylgard 184 Batch A was investigated by Flowers and Switzer ${ }^{23}$ through the additional reaction of iodine monochloride in glacial acetic acid. According to the report, the vinyl concentration of Batch A was equal to $2.2 \cdot 10^{-4} \mathrm{~mol} / \mathrm{g}$. Thus, $0.5 \mathrm{~g}$ of PDMS microspheres should have molar numbers of vinyl groups less than $1.1 \cdot 10^{-4} \mathrm{~mol}$. In the titration experiment, $2.5 \mathrm{~g}$ of mono-functional hydride, which has a molar number of hydrides at approximately $5.5 \cdot 10^{-4} \mathrm{~mol}$, was used to ensure excess monofunctional hydride groups. The mixture containing $0.5 \mathrm{~g}$ of PDMS microspheres and $2.5 \mathrm{~g}$ of mono-functional hydride was reacted for 24 hours at $80^{\circ} \mathrm{C}$ in the presence of a 50ppm catalyst. Unreacted mono-functional hydride and a soluble fraction were removed by swelling with heptane for 48 hours, after which the titrated PDMS microspheres were separated from the heptane solution. The residual heptane was removed by evaporation over 48 hours under atmospheric conditions. Afterwards, the titrated PDMS microspheres were weighed and their surface vinyl concentration was calculated.

\section{Results and discussion}

\section{Size distribution of vinyl functional PDMS microspheres}

The diameter of 10:1 (reference sample), 15:1, 20:1 and 25:1 PDMS microspheres fell between 10 $\mu \mathrm{m}$ to $500 \mu \mathrm{m}$, indicating that highly polydisperse PDMS microspheres were obtained (Figure 1). It is evident that the mixing ratio did not affect the mean diameter of the PDMS microspheres, which can be attributed to the mean diameter being determined mainly by the stirring rate of the impeller and the resulting energy transferred to the suspension medium ${ }^{24}$. On the other hand, the PDMS microspheres displayed broader size distribution along with an increasing mixing ratio, because the viscous PDMS suspension, which arose from the high mixing ratio of high viscosity vinyl-terminated PDMS to a low viscosity crosslinker, is hard to disperse and thus yields PDMS microspheres with broad size distribution ${ }^{25}$. 


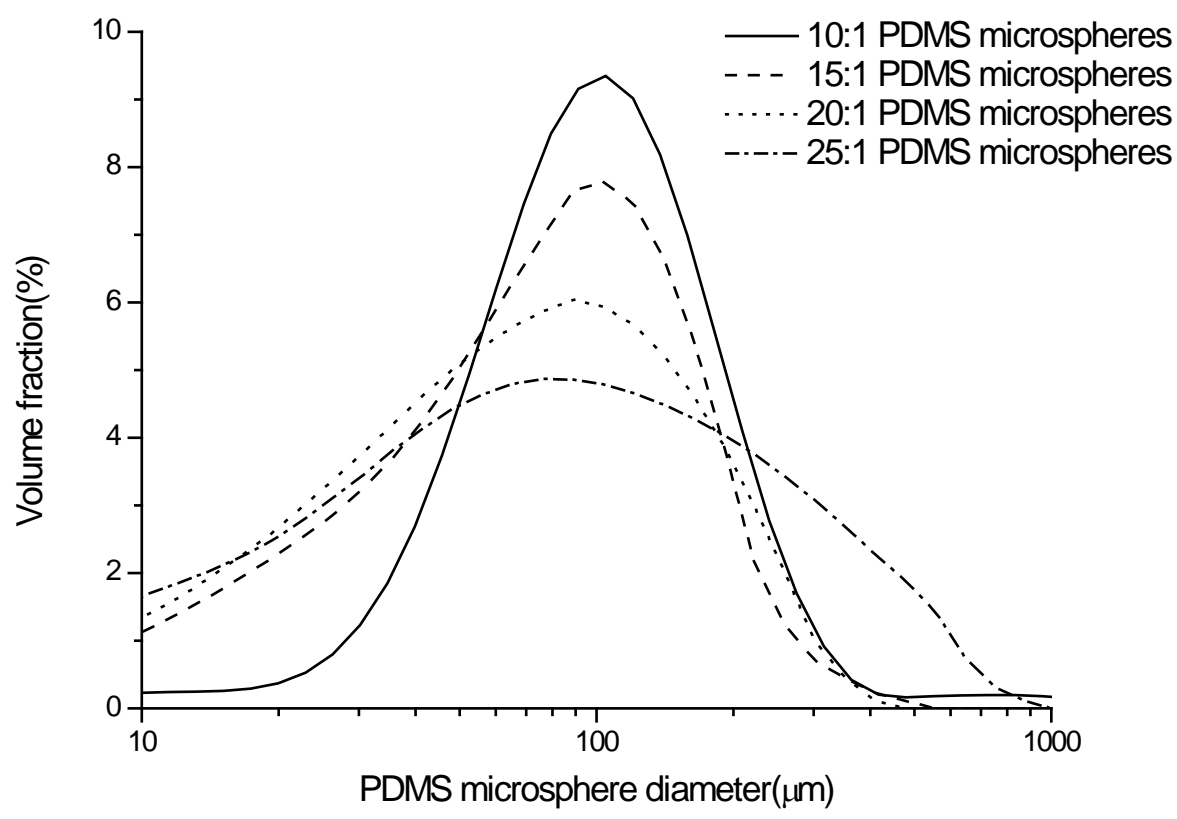

Figure 1. Size distribution of 10:1 (reference sample), 15:1, 20:1 and 25:1 PDMS microspheres prepared from emulsion polymerisation.

\section{Soluble fraction of vinyl functional PDMS microspheres}

In the preparation of vinyl functional PDMS microspheres, unreacted PDMS was trapped in the network due to its physical interaction with elastically active strands. Upon the swelling of the PDMS microspheres in heptane, the physical interaction decreased significantly and the unreacted PDMS was dissolved in heptane as a soluble fraction.

To determine the soluble fraction of vinyl functional PDMS microspheres, the PDMS microspheres were swelled in heptane for 48 hours. After the swelling procedure, they were dried at an ambient temperature for another 48 hours, to remove the residual heptane, and then weighed. The soluble fraction of the PDMS microspheres was calculated from the following equation:

$$
w_{\text {sol }} \%=\frac{m_{1}-m_{2}}{m_{1}} \cdot 100 \%
$$

where $m_{1}$ is the weight of vinyl functional PDMS microspheres before swelling in heptane, $m_{2}$ is the weight of vinyl functional PDMS microspheres after swelling in heptane and drying and $w_{\text {sol }} \%$ is the fraction of solubles.

The soluble fraction of the PDMS microspheres was lower than $17 \%$ in all samples (Table 1 ), indicating that they were highly crosslinked despite the nonstoichiometric conditions. Meanwhile, this figure increased 
from $7 \%$ to $16 \%$ when the mixing ratio increased from $15: 1$ to $25: 1$, which can be attributed to the amount of unreacted PDMS in the microspheres increasing with the increase in the mixing ratio.

PDMS microspheres have a larger surface area and a higher amount of surface defects than pure PDMS elastomer. Thus, their soluble fraction should not be compared with that of the pure PDMS elastomer, which will be discussed in detail later.

\section{Surface vinyl concentration of PDMS microspheres}

The surface vinyl concentration of PDMS microspheres was analysed by titration with mono-functional hydride. Two types of surface vinyl concentration, namely surface vinyl concentration by weight $\left(c_{\text {vinyl }}(w t)\right)$ and surface vinyl concentration by area $\left(c_{\text {vinyy }}(\right.$ surface $\left.)\right)$, are defined as follows:

$$
\begin{gathered}
c_{\text {vinyl }}(w t)=\frac{n_{\text {vinyl }}}{m_{3}} \\
c_{\text {vinyl }}(\text { surface })=\frac{n_{\text {vinyl }}}{A_{P M}}
\end{gathered}
$$

where $n_{\text {vinyl }}$ and $A_{P M}$ are the molar number and the surface area of PDMS microspheres, respectively, and $m_{3}$ is the mass of PDMS microspheres before titration. In order to estimate the surface area of the PDMS microspheres, see Supplementary Information part 1. Since mono-functional hydride was reacted with vinyl at a molar ratio of 1:1 via hydrosilylation reaction, the molar number of reacted mono-functional hydrides $\left(n_{\text {hydride }}\right)$ should equal the molar number of vinyl groups. Thus, we have:

$$
n_{\text {vinyl }}=n_{\text {hydride }}=\frac{m_{\text {hydride }}}{M_{\text {hydride }}}
$$

where $m_{\text {hydride }}$ and $M_{\text {hydride }}$ are the mass and the molecular weight of the mono-functional hydride, respectively. Upon the accomplishment of titration, titrated PDMS microspheres were swollen in heptane to remove unreacted mono-functional hydride and the soluble fraction, the latter of which should therefore be subtracted from the weight of the PDMS microspheres in the calculation. Thus, we obtain

$$
n_{\text {vinyl }}=\frac{m_{4}-m_{3} \cdot\left(1-w_{\text {sol }} \%\right)}{M_{\text {hydride }}}
$$

where $m_{4}$ is the mass of PDMS microspheres after reacting with the mono-functional hydride and $w_{\text {sol }} \%$ is the soluble fraction of the PDMS microspheres.

During titration, the swelling of PDMS microspheres was limited due to their highly crosslinked structure. Thus, the titration method ensured that only surface vinyl groups which were accessible for further crosslinking reaction would react with the mono-functional hydride. The surface vinyl concentrations of the 
15:1, 20:1 and 25:1 PDMS microspheres are summarised in Table 2. Surface vinyl concentration increased in line with the mixing ratio due to a larger amount of unreacted vinyl on the surface of the PDMS microspheres.

The ratios of surface vinyl to total vinyl concentrations are also summarised in Table 2 . In order to estimate total vinyl concentration, see Supplementary Information part 2. The ratio of surface vinyl to total vinyl concentrations ranged from $58 \%$ to $78 \%$, indicating that the vinyl groups were located primarily on the surface of the PDMS microspheres. Such a high fraction arises from the low efficiency of the crosslinking reaction on the surface of the PDMS microspheres in the preparation, because it was unfavourable at the aqueous interface and PDMS is immiscible in water ${ }^{26}$. The high surface concentration of vinyl groups allows for further functionalization of the microspheres or for further crosslinking of the microspheres as investigated in the following.

\section{Crosslinking reaction between PDMS microspheres and a hydride crosslinker}

To evaluate the crosslinking reaction between PDMS microspheres and a hydride crosslinker, a typical mixture containing a $1 \mathrm{~g}$ PDMS microsphere, a hydride crosslinker and $0.5 \mathrm{~g}$ non-reactive silicone oil was made. The required amount of hydride crosslinker was estimated from the surface vinyl concentration of the PDMS microspheres based on stoichiometric imbalance $r$, calculated from:

$$
r=\frac{n_{\text {hydride }}}{n_{\text {vinyl }}}=\frac{\frac{m_{H M S-301}}{M_{H M S-301}} \cdot f_{H M S-301}}{m_{P M} \cdot c_{\text {vinyl }}(w t)}
$$

where $n$ is the number of moles, $m$ is mass, $M$ is molecular weight, $f$ is the functionality of the hydride crosslinker and $c$ is surface vinyl concentration by weight, and where subscripts HMS-301 and PM denote the hydride crosslinker and vinyl functional PDMS microsphere, respectively.

The mixture containing the PDMS microspheres, hydride crosslinker and non-reactive silicone oil was cured at $50^{\circ} \mathrm{C}$ for 5 hours. Figure 2a shows the microspheres before the crosslinking reaction. It is evident that they aggregated due to inter-particle physical interaction and remained spherical due to the lack of potential chemical crosslinking points, which in turn allowed them to be separated again under shear force or swelling. In contrast, Figure $2 \mathrm{~b}$ shows that a macroscopically crosslinked network was obtained and the PDMS microspheres were deformed after the crosslinking reaction. This indicates an extensive crosslinking reaction between the PDMS microspheres and the hydride crosslinker. After cooling down to room temperature, the macroscopic PDMS network remained in its gel state, indicating that gelation did not form from the physical association between the PDMS microspheres. 

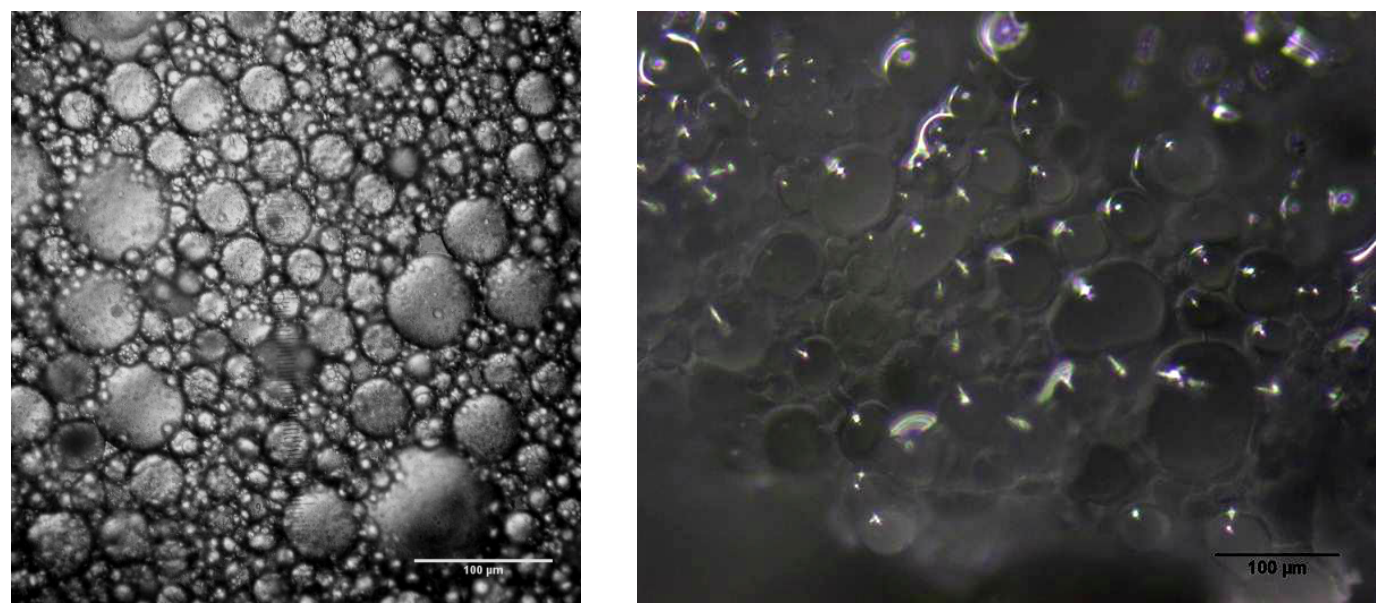

Figure 2a(left), 2b(right). Representative microscopic images of 20:1 PDMS microspheres before (left) and after (right) a crosslinking reaction. The crosslinking reaction between 20:1 PDMS microspheres and the hydride crosslinker was performed at $50^{\circ} \mathrm{C}$ and at $r=5$ with $35 \%$ (wt) non-reactive silicone oil over a period of 5 hours.

The crosslinking reaction between PDMS microspheres and the hydride crosslinker was characterised quantitatively through rheological measurements. Stoichiometric balances/imbalances of 1, 3 and 5 were employed to investigate the influences of the amount of hydride crosslinker on the crosslinking reaction. High stoichiometric imbalances (e.g. $r=5$ ) were used in the crosslinking reaction for the following reasons: firstly, some of the hydride crosslinker might be absorbed by the PDMS microspheres and then react with the vinyl groups in the bulk of the PDMS microspheres, resulting in increasing the crosslinking density of the bulk of the PDMS microspheres without yielding inter-particle crosslinking points. Secondly, the hydride crosslinker might react with surface vinyl groups from the same PDMS microsphere rather than yielding inter-particle crosslinking points. This would lead to increasing the surface crosslinking density of the PDMS microspheres rather than yielding a macroscopic network.

For facile crosslinking between PDMS microspheres and the hydride crosslinker, 10ppm platinum catalyst was added to the system. The concentration of this catalyst was much higher than for the residual platinum catalyst on the surface of the PDMS microspheres resulting from the microsphere production step. Consequently, the concentration of platinum catalyst in each system was ensured to be similar and the crosslinking reaction rate should not be significantly affected by concentration variations.

The storage moduli of the mixtures containing PDMS microspheres, the hydride crosslinker and nonreactive silicone oil vary in line with small changes in the volume fraction of components ${ }^{27}$. Hence, the storage moduli of the mixtures were normalised by dividing by the initial storage modulus $\left(G^{\prime}(0)\right)$ of the respective mixture ${ }^{28}$. The absolute moduli are given in Supplementary Info 3. An exact determination of the progress of the curing reaction could be performed by detection of the disappearance of vinyl groups by e.g. spectroscopy.

Figure 3 shows the evolution of the normalised storage moduli as a function of time, with $r=1$ and $r=5$ ( $r=3$ not shown for clarity, but the results follow an identical trend as $r=1$ and $r=5$ and falls in between). The curve of the normalised storage modulus of 15:1 PDMS microspheres at $r=5$ exhibits a steeper slope than that at $r=1$, indicating that the crosslinking reaction rate increased in line with the amount of hydride 
crosslinker. Meanwhile, the curves of the storage moduli for 20:1 and 25:1 PDMS microspheres show a steeper slope than for the 15:1 PDMS microspheres at identical stoichiometric imbalances, thus indicating that the crosslinking reaction rate also increased in line with surface vinyl concentration.

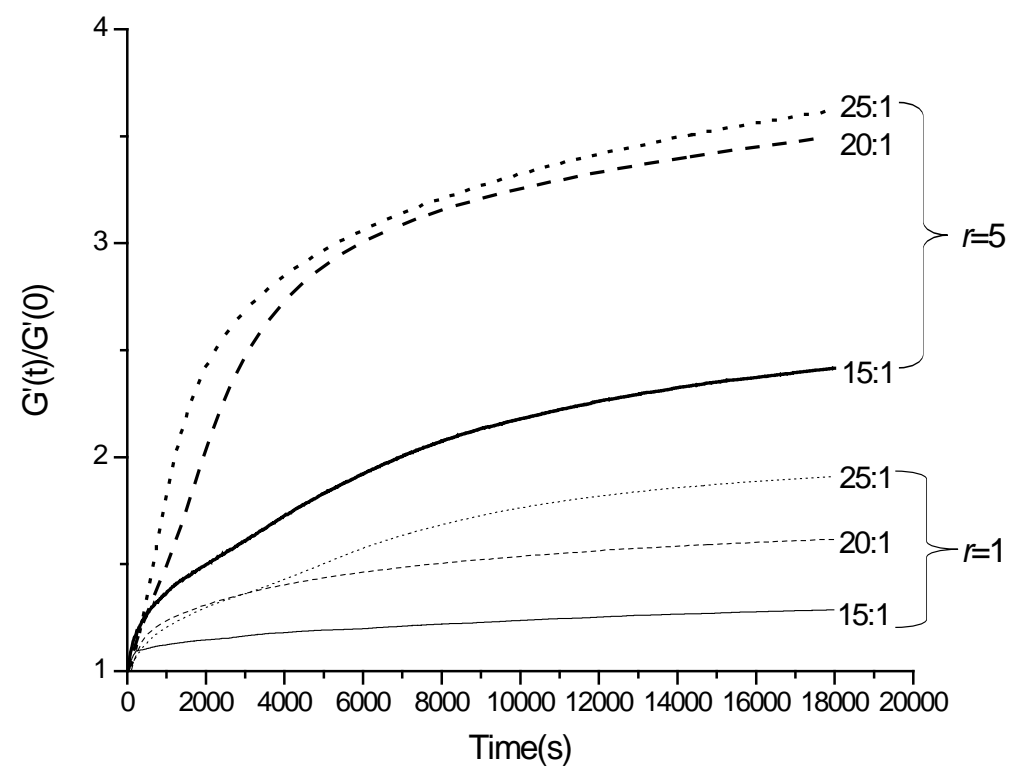

Figure 3. Normalised storage moduli of mixtures containing PDMS microspheres, hydride crosslinker and non-reactive silicone oil $(35 \%(w t))$ over a period of 5 hours at $50^{\circ} \mathrm{C}$. The measurements were performed with a strain of $2 \%$ and a frequency of $1 \mathrm{~Hz}$.

The crosslinking reaction between 20:1 PDMS microspheres and the hydride crosslinker at $r=1$ and $r=5$ was further evaluated over a period of 72 hours. The normalised storage moduli of the resulting macroscopic PDMS networks obtained at 5 hours, 24 hours, 48 hours and 72 hours are listed in Table 3 . The values of $G^{\prime}(24 h) / G^{\prime}(0), G^{\prime}(48 h) / G^{\prime}(0)$ and $G^{\prime}(72 h) / G^{\prime}(0)$ were closely matched, indicating that $G^{\prime}(72 h)$ could be regarded as the equilibrium storage modulus of the macroscopic network. Meanwhile, the ratio between $G^{\prime}(5 h)$ and $G^{\prime}(72 h)$ was higher than 0.95 , suggesting that most crosslinking reactions finished within a period of 5 hours $^{29}$. Thus, it should be reasonable to use the storage modulus obtained after reacting for 5 hours, in order to describe the crosslinking reaction between PDMS microspheres and the hydride crosslinker. The storage moduli of the resulting macroscopic network obtained at 5 hours are denoted as terminal storage moduli.

Normalised terminal storage moduli $\left(G^{\prime}(5 h) / G^{\prime}(0)\right)$ are obtained from Figure 3 and shown in Figure 4 to compare the stiffness of the obtained macroscopic PDMS networks. The normalised terminal storage modulus increased in line with stoichiometric imbalance in $1<r<5$, indicating that a stronger macroscopic PDMS network was obtained in the presence of an increasing amount of hydride crosslinker. Meanwhile, the normalised terminal storage modulus also increased in line with the mixing ratio, i.e. the macroscopic PDMS networks obtained from 20:1 and 25:1 PDMS microspheres exhibited higher normalised terminal storage moduli than obtained from 15:1 PDMS microspheres at $r=5^{30}$. This can be attributed to the surface 
vinyl concentration of the 20:1 and 25:1 PDMS microspheres being higher than that of their 15:1 counterparts.

The initial storage moduli of 20:1 and 25:1 PDMS microspheres, as well as the terminal storage moduli, were similar. Thus, the 20:1 PDMS microspheres were selected to investigate the influence of stoichiometric imbalance across a wider range and the weight fraction of non-reactive silicone oil on the terminal storage modulus of the obtained macroscopic PDMS network.

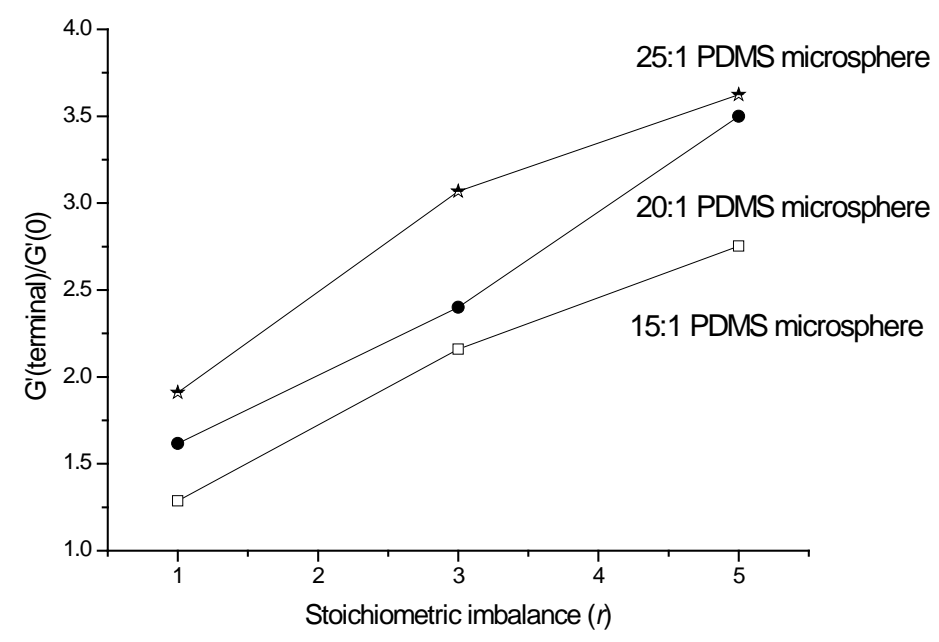

Figure 4. Normalised terminal storage moduli of the macroscopic PDMS network, obtained from the crosslinking reaction between 15:1, 20:1 and 25:1 PDMS microspheres, the hydride crosslinker and nonreactive silicone oil (35\% (wt)) as a function of $r$. (Solid lines are a visual guide only).

Influence of stoichiometric imbalance and the weight fraction of non-reactive silicone oil on the terminal storage moduli of a macroscopic PDMS network

In this section, the terminal storage moduli of the obtained macroscopic PDMS network were investigated across a wider stoichiometric imbalance $(1<r<11)$ range. Similar to the previous section, the terminal storage moduli were also normalised by dividing the initial storage moduli of respective mixtures. A maximum normalised terminal storage modulus was obtained at $r=7$ (Figure 5), after which any excess hydride crosslinker did not increase the crosslinking density of the macroscopic network. The reasons for obtaining a maximum normalised terminal storage modulus at high stoichiometric imbalances are explained as follows: firstly, part of the hydride crosslinker was absorbed by the PDMS microspheres and then reacted with the vinyl groups in the bulk of the PDMS microspheres. As a result, the absorbed hydride crosslinker only strengthened the PDMS microspheres and did not yield inter-particle crosslinking points. Secondly, a crosslinking reaction took place when the hydride crosslinker diffused to the surface vinyl groups attached to the PDMS microspheres. On completion of the crosslinking reaction, the hydride crosslinker was also covalently attached to the microspheres. Accordingly, some of the hydride groups in the crosslinker were unable to react with the surface vinyl groups due to a steric effect, resulting in the high amount of hydride crosslinker required to obtain the maximum terminal storage modulus. 


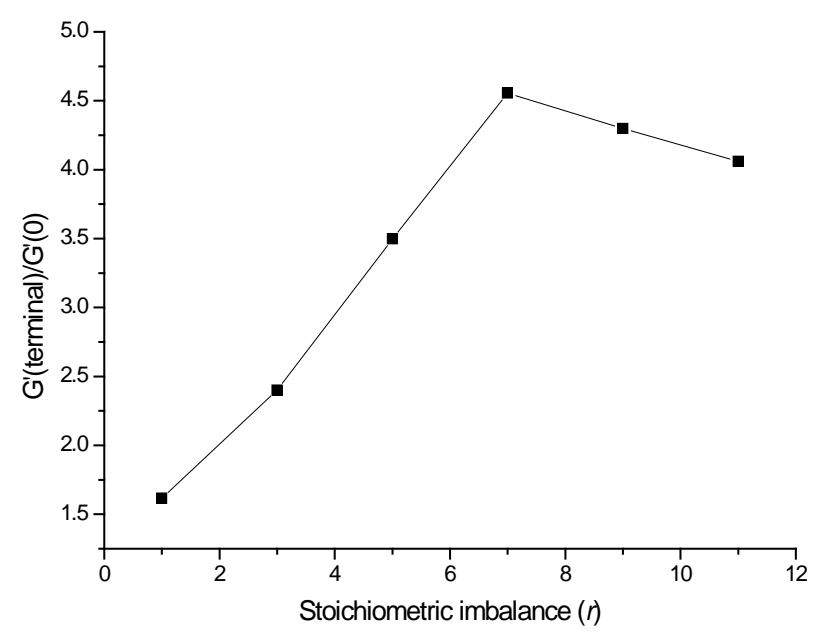

Figure 5. Normalised terminal storage moduli of a macroscopic PDMS network obtained from the crosslinking reaction between 20:1 PDMS microspheres, the hydride crosslinker and non-reactive silicone oil (35\% (wt)) as a function of $r$ ranging from 1 to 11 . (Solid lines are a visual guide only).

To investigate the influence of the weight fraction of non-reactive silicone oil on the terminal storage modulus of a macroscopic PDMS network, silicone oil with a molecular weight of $2,000 \mathrm{~g} / \mathrm{mol}$ was employed. The low molecular weight of the silicone oil ensured that it would not tangle with the macroscopic PDMS network. Consequently, the terminal storage modulus of the macroscopic PDMS network depended solely on the weight fraction of the silicone oil ${ }^{31}$. Similar to previous experiments, mixtures containing PDMS microspheres, a hydride crosslinker and non-reactive silicone oil were prepared. To ensure a sufficient amount of hydride crosslinker, high stoichiometric imbalances ( $r=5$ and $r=7)$ were used.

The normalised terminal storage moduli of the obtained macroscopic PDMS networks are plotted as a function of the weight fraction of silicone oil in Figure 6 . The trends of the normalised terminal storage modulus curves between $r=5$ and $r=7$ were similar, indicating that the silicone oil influenced the mechanical properties of the macroscopic networks in a similar manner in the presence of different amounts of hydride crosslinker. Meanwhile, the normalised terminal storage modulus increased in line with the weight fraction of the silicone oil in the range $15 \%$ (wt) to $35 \%$ (wt). This can be attributed to an increase in the weight fraction of the silicone oil, thereby enhancing the hydride crosslinker diffusion rate and thus yielding a stronger network. In the range $35 \%(\mathrm{wt})$ to $50 \%(\mathrm{wt})$, the normalised terminal storage modulus remained constant in line with an increase in the weight fraction of the silicone oil. This can be explained by the silicone oil promoting the diffusion rate of the crosslinker and diluting the polymer chains in the macroscopic PDMS network simultaneously. When the weight fraction of the silicone oil increased by up to 90\% $(\mathrm{wt})^{32}$, the PDMS microspheres in the mixture were diluted sufficiently to avoid inter-particle interactions. As a result, no crosslinking reaction between the PDMS microspheres took place and no macroscopic PDMS network could be obtained. 


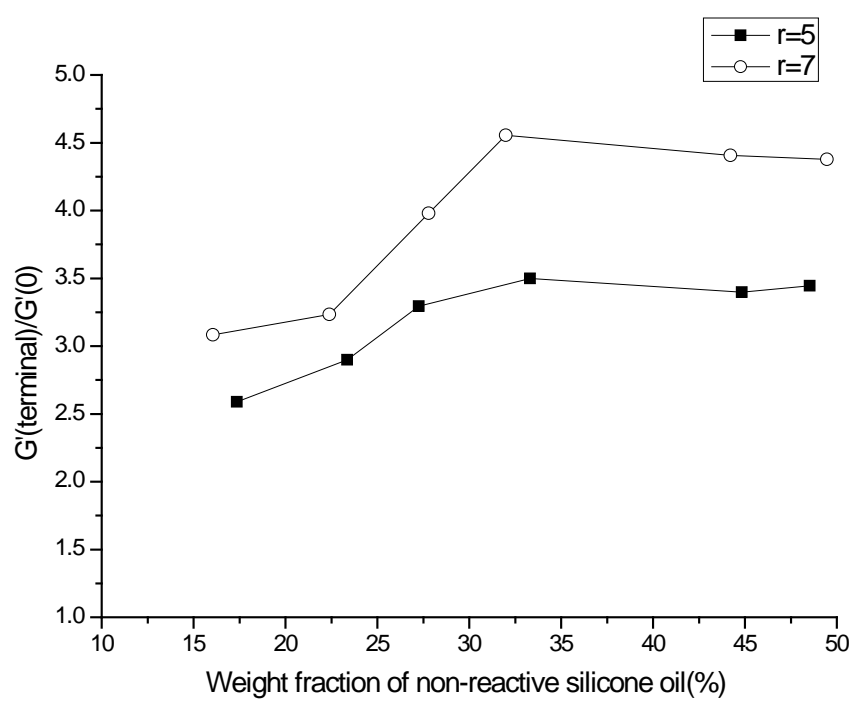

Figure 6. Normalised terminal storage moduli of a macroscopic PDMS network obtained from the crosslinking reaction between 20:1 PDMS microspheres, the hydride crosslinker and non-reactive silicone oil at $r=5$ and $r=7$ as a function of the weight fraction of silicone oil. (Solid lines are a visual guide only).

\section{Conclusions:}

A novel titration method, introduced for quantifying the surface vinyl concentration of PDMS microspheres, exploited the efficient and covalent bonding of mono-functional hydride to vinyl. The good reproducibility of the titration method implied that the titration method should be applicable to other commercial nonstoichiometric silicone materials where surface vinyl concentration is of importance. The surface vinyl groups of the PDMS microspheres allow for further crosslinking reactions with a hydride crosslinker in the presence of non-reactive silicone oil, thereby yielding a macroscopic PDMS network. The influence of stoichiometric imbalance, and the weight fraction of non-reactive silicone oil on the storage modulus of the macroscopic network, was investigated, and it was established that a maximum storage modulus ( 90kPa) can be obtained at $r=7$ and $35 \%(\mathrm{wt}$ ) of silicone oil.

A system containing PDMS microspheres, a hydride crosslinker and silicone oil could be used to introduce macroscopic PDMS elastomer to hard-to-reach places, for example fractured pipes in an oil field. In applications relevant to blocking fractures, the modulus of the macroscopic PDMS elastomer is essential to achieving blocking efficiency, as the macroscopic elastomer should withstand pressure differences during oil recovery. The optimum amount of hydride crosslinker and silicone oil used in the crosslinking reaction was found in the present study, which allows for designing an optimised system for blocking fractures. 
References:

(1) Kawaguchi, H. Prog. Polym. Sci. 2000, 25, 1171-1210.

(2) Jerschow, P. Silicone Elastomers; Springer: Shropshire, UK, 2001; Vol. 12.

(3) Uchida, T.; Mills, K. L.; Kuo, C. H.; Roh, W.; Tung, Y. C.; Garner, A. L.; Koide, K.; Thouless, M. D.; Takayama, S. Langmuir 2009, 25, 3102-3107.

(4) Ioppolo, T.; Otügen, M. V. Opt. Lett. 2010, 35, 2037-2039.

(5) Peng, S.; Zhang, M.; Niu, X.; Wen, W.; Sheng, P.; Liu, Z.; Shi, J. Appl. Phys. Lett. 2008, 92, 012108.

(6) González, L.; Ma, B.; Li, L.; Hansen, J. H.; Hvilsted, S.; Skov, A. L. Macromol. Mater. Eng. 2014, 299, 729-738.

(7) Larsen, A. L.; Hansen, K.; Hassager, O.; Bach, A.; Ndoni, S.; Jørgensen, M. Macromolecules 2003, 36, 10063-10070.

(8) Gaborieau, M.; Nebhani, L.; Graf, R.; Barner, L.; Barner-Kowollik, C. Macromolecules 2010, 43, 38683875.

(9) So, H.; Fawcett, A. S.; Sheardown, H.; Brook, M. A. J. Colloid Interface Sci. 2013, 390, 121-128.

(10) Downey, J.; Frank, R.; Li, W.; Stöver, H. Macromolecules 1999, 32, 2838-2844.

(11) Yamamoto, Y.; Okubo, M.; Iwasaki, Y. Colloid Polym. Sci. 1991, 269, 1126-1132.

(12) Madsen, F. B.; Dimitrov, I.; Daugaard, A. E.; Hvilsted, S.; Skov, A. L. Polym. Chem. 2013, 4, 17001707.

(13) Madsen, F. B.; Daugaard, A. E.; Fleury, C.; Hvilsted, S.; Skov, A. L. RSC Adv. 2014, 4, 6939-6945.

(14) Hu, X.; Zhou, J.; Zhang, N.; Tan, H.; Gao, C. J. Mech. Behav. Biomed. Mater. 2008, 1, 352-359.

(15) Fetters, L. J.; Lohse, D. J.; Milner, S. T.; Graessley, W. W. Macromolecules 1999, 32, 6847-6851.

(16) González, L.; Malgorzata, K.; Ma, B.; Li, L.; Hansen, J.H.; Hvilsted, S.; Skov, A. L. Macromol. Mater. Eng. 2014, 299, 1259-1267.

(17) Ma, B.; Hansen, J. H.; Hvilsted, S.; Skov, A. L. RSC Adv. 2014, 4, 47505-47512.

(18) Yao, C.; Lei, G.; Li, L.; Gao, X. Energy \& Fuels 2012, 26, 5092-5101.

(19) Bejenariu, A. G.; Yu, L.; Skov, A. L. Soft Matter 2012, 8, 3917-3923.

(20) Westhaus, E.; Messersmith, P. B. Biomaterials 2001, 22, 453-462. 
(21) Sanborn, T. J.; Messersmith, P. B.; Barron, A. E. Biomaterials 2002, 23, 2703-2710.

(22) Frankær, S. M. G.; Jensen, M. K.; Bejenariu, A. G.; Skov, A. L. Rheol. Acta 2012, 51, 559-567.

(23) Flowers, G.; Switzer, S. Background material properties of selected silicone potting compounds and raw materials for their substitutes; Dow Corning report; 1978; pp. 1-19.

(24) Hinze, J. AIChE J. 1955, 1, 289-295.

(25) Denkbaş, E.; Kiliçay, E.; Birlikseven, C.; Ozturk, E. React. Funct. Polym. 2002, 50, 225-232.

(26) Marcus, L. Organic Reactions in Water; Blackwell: Oxford, UK, 2007; pp. 211-221.

(27) Gisler, T.; Weitz, D. A. Phys. Rev. Lett. 1999, 82, 1606-1609.

(28) Trappe, V.; Weitz, D. Phys. Rev. Lett. 2000, 85, 449-452.

(29) Bonino, C. A.; Samorezov, J. E.; Jeon, O.; Alsberg, E.; Khan, S. A. Soft Matter 2011, 7, 11510-11517.

(30) Chambon, F.; Winter, H. H. J. Rheol. 1987, 31, 683-697.

(31) Mrozek, R. A.; Cole, P. J.; Otim, K. J.; Shull, K. R.; Lenhart, J. L. Polymer 2011, 52, 3422-3430.

(32) Valette, L.; Pascault, J. P.; Magny, B. Macromol. Mater. Eng. 2002, 287, 41-51.

(33) Colas, A. Inorganic polymers; Nova Science: Seneffe, Belgium, 1994; Vol. 38, pp. 656-659. 
List of tables:

Table 1. Soluble fraction of vinyl functional PDMS microspheres from different mixing ratios

\begin{tabular}{|l|l|l|l|}
\hline Sample ID & $\begin{array}{l}\text { Weight of PDMS } \\
\text { microspheres }\left(m_{1} \mathrm{~g}\right)\end{array}$ & $\begin{array}{l}\text { Weight of PDMS microspheres } \\
\left(m_{2} \mathrm{~g}\right) \text { after swelling in heptane }\end{array}$ & $\begin{array}{l}\text { Soluble fraction of PDMS } \\
\text { microspheres }\left(w_{\text {sol }} \%\right)\end{array}$ \\
\hline $\begin{array}{l}15: 1 \quad \text { PDMS } \\
\text { microspheres }\end{array}$ & $0.55 \pm 0.03$ & $0.51 \pm 0.03$ & $7 \pm 1$ \\
\hline $\begin{array}{l}20: 1 \quad \text { PDMS } \\
\text { microspheres }\end{array}$ & $0.53 \pm 0.02$ & $0.48 \pm 0.02$ & $9.3 \pm 0.2$ \\
\hline $\begin{array}{l}25: 1 \text { PDMS } \\
\text { microspheres }\end{array}$ & $0.57 \pm 0.03$ & $0.47 \pm 0.01$ & $16 \pm 3$ \\
\hline
\end{tabular}

The measurements were averaged from 3-4 samples.

Table 2. Surface vinyl concentration of PDMS microspheres from different mixing ratios

\begin{tabular}{|c|c|c|c|c|c|c|}
\hline Sample ID & $\begin{array}{l}\text { Weight of } \\
\text { PDMS } \\
\text { microspheres } \\
\left(\mathrm{m}_{3} \mathrm{~g}\right)\end{array}$ & 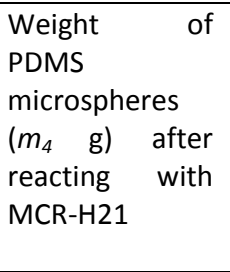 & $\begin{array}{l}\text { Surface vinyl } \\
\text { concentration } \\
\left(c_{\text {vinyl }}(w t)\right) \\
\text { PDMS of } \\
\text { microspheres by } \\
\text { weight }\left(10^{-5}\right. \\
\text { mol/g)* }\end{array}$ & $\begin{array}{l}\text { Surface vinyl } \\
\text { concentration } \\
\left(c_{\text {vinyl }}(\text { sur })\right) \\
\text { PDMS of } \\
\text { microspheres by } \\
\text { surface area }\left(10^{-3}\right. \\
\left.\mathrm{mol} / \mathrm{m}^{2}\right)^{*}\end{array}$ & $\begin{array}{l}\text { Total vinyl } \\
\text { concentration } \\
\text { of PDMS } \\
\text { microspheres } \\
\left(10^{-5} \mathrm{~mol} / \mathrm{g}\right)\end{array}$ & $\begin{array}{l}\text { The ratio of } \\
\text { surface vinyl to } \\
\text { total vinyl } \\
\text { concentrations }\end{array}$ \\
\hline $\begin{array}{l}\text { 15:1 PDMS } \\
\text { microspheres }\end{array}$ & $0.62 \pm 0.06$ & $0.57 \pm 0.05$ & $0.7 \pm 0.2$ & $0.6 \pm 0.2$ & 1.2 & 58 \\
\hline $\begin{array}{l}\text { 20:1 PDMS } \\
\text { microspheres }\end{array}$ & $0.48 \pm 0.01$ & $0.50 \pm 0.01$ & $2.4 \pm 0.1$ & $2.0 \pm 0.1$ & 3.2 & 75 \\
\hline $\begin{array}{l}\text { 25:1 PDMS } \\
\text { microspheres }\end{array}$ & $0.53 \pm 0.05$ & $0.53 \pm 0.05$ & $3.4 \pm 0.4$ & $2.9 \pm 0.3$ & 4.4 & 78 \\
\hline
\end{tabular}

*The measurements were averaged from 3-4 samples.

Table 3. Normalised storage moduli of mixtures containing 20:1 PDMS microspheres, a hydride crosslinker and non-reactive silicone oil (35\% (wt)) obtained after reacting for 5 hours, 24 hours, 48 hours and 72 hours at $50^{\circ} \mathrm{C}$. The measurements were performed with a strain of $2 \%$ and a frequency of $1 \mathrm{~Hz}$.

\begin{tabular}{|l|l|l|l|l|}
\hline & $G^{\prime}(5 h) / G^{\prime}(0)$ & $G^{\prime}(24 h) / G^{\prime}(0)$ & $G^{\prime}(48 h) / G^{\prime}(0)$ & $G^{\prime}(72 h) / G^{\prime}(0)$ \\
\hline Normalised storage modulus at $r=1$ & 1.61 & 1.63 & 1.63 & 1.63 \\
\hline Normalised storage modulus at $r=5$ & 3.49 & 3.61 & 3.63 & 3.64 \\
\hline
\end{tabular}

